\title{
M. Menière: intratympanale Gabe von Methylprednisolon so wirksam wie Gentamicin
}

\begin{abstract}
Ein klassischer Therapieansatz bei Morbus Menière ist die intratympanale Gabe von Gentamicin. Dies führt dosisabhängig zu einem Untergang der vestibulären Rezeptoren und damit zu einem Ausfall der vestibulären Funktionen. Bei etwa $20 \%$ der so behandelten Patienten kommt es auch zu einer Hörminderung oder sogar zu einem Hörverlust. Daher ist es dringend notwendig, andere Therapien zu entwickeln, bei denen die vestibulären Funktionen intakt bleiben und insbesondere kein Risiko für eine Hörminderung besteht.
\end{abstract}

\begin{abstract}
ᄃ ine doppelblinde Therapiestudie bei
C Patienten im Alter zwischen 18 und

70 Jahren und therapierefraktärer unilateraler Menièrscher Erkrankung ging der Frage nach, ob sich die intratympanale Gabe von Methylprednisolon bei der Behandlung des Morbus Menière möglicherweise als genauso wirksam erweist wie die etablierte Therapie mit Gentamicin. Die Studienteilnehmer erhielten entweder zwei intratympanale
\end{abstract}

Injektionen mit $62,5 \mathrm{mg} / \mathrm{ml}$ Methylprednisolon oder $40 \mathrm{mg} / \mathrm{ml}$ Gentamicin im Abstand von zwei Wochen. Die Beobachtungszeit betrug zwei Jahre. Der primäre Studienendpunkt war die Häufigkeit von Schwindelattacken über die letzten sechs Monate der Beobachtungszeit gegenüber den sechs Monaten vor der ersten Therapie.

Insgesamt wurden 60 Patienten randomisiert. In der Gentamicin-Gruppe kam es zu einem Rückgang der Schwindelattacken von 19,9 Anfällen in den sechs Monaten vor Therapie auf 2,5 in den letzten sechs Monaten der Beobachtungszeit. Dies entspricht einer Reduktion von $87 \%$. In der Methylprednisolon-Gruppe kam es zu einem Rückgang der Schwindelattacken von 16,4 auf 1,6, was einer Reduktion um $90 \%$ entspricht. Die Behandlung wurde sehr gut vertragen.

Fazit: Die intratympanale Gabe von Methylprednisolon ist bei der Menièrschen Erkrankung ebenso wirksam wie die Gabe von Gentamicin.

red

Patel M, Agarwal K, Arshad Q et al. Intratympanic methylprednisolone versus gentamicin in patients with unilateral Ménière's disease: a randomised, double-blind, comparative effectiveness trial. Lancet 2016; 388: 2753-62

\section{Chronische Rhinosinusitis: erhöhte Revisionsrate nach Sinus-Op bei Polypennachweis}

Kanadische Ärzte um Dr. Luke Rudmik von der Universität von Calgary sind in einer retrospektiven Studie der Frage nachgegangen, wie sich bei Patienten mit chronischer Rhinosinusitis die Ergebnisse einer endoskopischen SinusChirurgie (ESS, endoscopic sinus surgery) durch Ärzte an Kliniken in Alberta unterscheiden. Primärer Studienendpunkt war die Revisionsrate fünf Jahre nach dem Eingriff.

F ür die Studie standen die Daten von 43 Chirurgen zur Verfügung, die bei 2.168 Patienten mit chronischer Rhinosinusitis eine ESS vorgenommen hatten. Wie Rudmik und seine Kollegen berichten, hatte jeder Studienteilnehmer in einem Zeitraum von drei Jahren durchschnittlich 50 Eingriffe vorgenommen (mindestens 16 und maximal 167). Fünf Jahre nach dem Primäreingriff mussten sich 239 Patienten einem Revisionseingriff unterziehen. Die Revisionsrate nach diesem Zeitraum berechnete sich zu durchschnittlich 10,6\% (zwischen $2,4 \%$ und $28,6 \%$ ). Dabei wurde bei zwei Chirurgen (5\%) eine niedrigere Revisionsrate als erwartet ( $1,8 \%$ und $3,1 \%)$, bei sieben Chirurgen (16\%) dagegen eine schlechtere dokumentiert (zwischen $17,7 \%$ und 28,6\%). Nach Ansicht der Studienärzte lässt sich somit die chirurgische Versorgung noch verbessern.

Die Kollegen um Rudmik prüften darüber hinaus, welche von 24 Parametern - etwa Diabetes, Asthma, Adipositas oder nasale Polypen - mit der Fünf-JahresRevisionsrate assoziiert sind. Bei der adjustierten Berechnung wurden $\mathrm{p}$-Werte $\leq 0,002$ als signifikant definiert. Nur drei Parameter kristallisierten sich schließlich dabei heraus. So waren das Vorhandensein nasaler Polypen und die vermehrte systemische Applikation von Kortikosteroiden vor dem endoskopischen Eingriff mit einer erhöhten Wahrscheinlichkeit für eine Revisions-Op. as- soziiert. Das Risiko für eine Revision war bei Nachweis von Polypen verdoppelt (Odds Ratio [OR]: 2,07; $95 \%$-Konfidenzintervall zwischen 1,59 und 2,70) und bei vermehrter Steroidtherapie um ein Drittel erhöht (OR: 1,33; 95 \%-Konfidenzintervall zwischen 1,19 und 1,48). Im Gegensatz dazu war eine gleichzeitige Septumplastik mit einem um 30\% niedrigeren Revisionsrisiko assoziiert (OR: 0,70; 95\%-Konfidenzintervall zwischen 0,55 und 0,89$)$..

Fazit: Bei Patienten mit chronischer Rhinosinusitis sind nach endoskopischer $\mathrm{Si}$ nus-Chirurgie möglicherweise Nasenpolypen und häufige systemische Kortikoidanwendung mit einer erhöhten Revisionsrate assoziert. Einschränkend weisen Rudmik und seine Kollegen aber darauf hin, dass aufgrund der Datenart eine genaue Risikoabschätzung nicht möglich gewesen sei. Zudem sei unklar, wie die Revisionsraten außerhalb von Alberta sind.

Peter Leiner

Rudmik L et al. Evaluating Surgeon-Specific Performance for Endoscopic Sinus Surgery. JAMA Otolaryngol Head Neck Surg 2017, online 22. Juni 